\title{
Erratum
}

\section{Adefovir dipivoxil monotherapy is a sufficiently effective treatment modality for Japanese patients with lamivudine-resistant chronic hepatitis B}

Ken Sato, Hitoshi Takagi, Satoru Kakizaki, Naondo Sohara, and Masatomo Mori

Department of Medicine and Molecular Science, Gunma University Graduate School of Medicine, 3-39-15 Showa, Maebashi 371-8511, Japan

J Gastroenterol 2006; 41:283-285

Note

Case 5 in Table 1 should be "Male", not "Female". 